\title{
Online monitoring and diagnosis of high voltage circuit breaker faults: feature extraction analysis of vibration signals
}

\author{
Long $\mathrm{Li}^{*}$, Jianfeng Xiao, Bin Wu, Mengge Zhou, and Qian Wang \\ Electric Power Research Institute, State Grid Chongqing Electric Power Company, Chongqing 401123, PR China
}

Received: 19 September 2019 / Accepted: 17 October 2019

\begin{abstract}
The development of power grid system not only increases voltage and capacity, but also increases power risk. This paper briefly introduces the feature extraction method of the vibration signal of high voltage circuit breaker and support vector machine (SVM) algorithm and then analyzed the high voltage circuit breaker in three states: normal operation, fixed screw loosening and falling of opening spring, using the SVM based on the above feature extraction method. The results showed that the accuracy and precision rates of fault identification of circuit breaker were the highest by using the wavelet packet energy entropy extraction features, the false alarm rate was the lowest, and the detection time was the shortest.
\end{abstract}

Keywords: High voltage circuit breaker / vibration signal / feature extraction / wavelet packet energy entropy

\section{Introduction}

With the rapid development of economic construction, the national demand for electricity is increasing, and the voltage and capacitance of power plants are also increasing rapidly [1]. Moreover, higher requirements are put forward for the safe operation of power plants. For example, when the high-voltage transmission line fails, the fault area is quickly isolated from and the normal area to ensure the overall safety of the power grid. High-voltage circuit breaker is one of the power system protection devices. Its working principle of realizing the isolation of fault and nonfault lines in power grid is that the energy provided by the operating mechanism is transferred to the interrupting element by driving rod when the probe sensing system fails, and the interrupting element realizes the opening and closing of the circuit by the transferred energy. Once the high voltage circuit breaker breaks down in the power operation, the loss will be enormous [2]. Huang et al. [3] proposed a mechanical fault diagnosis method for high voltage circuit breaker based on wavelet time-frequency entropy and one-class support vector machine (SVM), optimized the parameters using particle swarm algorithm, and verified the accuracy of the method through experiments. Yang et al. [4] proposed a fault diagnosis method based on probabilistic neural network and found through simulation that the diagnosis model converged fast, had high accuracy and was easy to train. Bing et al. [5] removed noise from circuit breaker vibration signal using soft

\footnotetext{
* Corresponding author: longl_li@yeah.net
}

threshold method, then extracted vibration characteristics using wavelet transform and time-frequency entropy, and identified faults using generalized regression neural network. The experimental results showed that the method could effectively identify fault types. Huang et al. [6] proposed a mechanical fault diagnosis method based on local mean decomposition and time-division energy entropy, which combined SVM description with fuzzy c-means clustering. The experimental results showed that the method was effective for both known faults with training samples and unknown faults without training samples. Kong et al. [7] judged faults using the electromagnetic pulse generated by partial discharge when insulation fault of circuit breaker appeared and verified by experiments that the circuit breaker insulation fault could be distinguished by the characteristics of electromagnetic pulse waveform. This paper briefly introduced the feature extraction method of the vibration signal of high voltage circuit breaker and SVM algorithm and analyzed the high voltage circuit breaker in states of normal operation, fixed screw loosening and falling of opening spring using the SVM algorithm based on the above feature extraction method.

\section{Feature extraction}

The maintenance of high voltage circuit breakers has undergone the development process of break maintenance, planned maintenance and condition maintenance. The break maintenance means repairing passively after the breakdown due to the immature technology, which not only reacts slowly, but also can cause loss easily when 


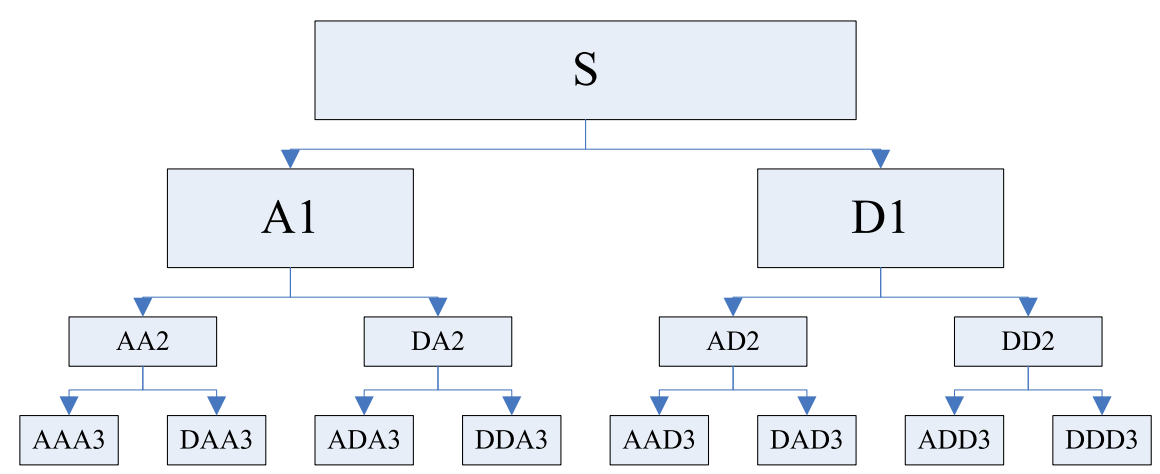

Fig. 1. Diagram of wavelet packet decomposition.

repairing. The planned maintenance is to repair the circuit breakers regularly. Although fault prevention was actively, circuit breakers need to be disassembled, which costs high and may cause damages in the process of disassembly. Condition maintenance is to intelligently determine whether the circuit break fails or not through the feature parameters under different conditions using sensor and computer technology. In this paper, the mechanical vibration characteristics in the operation of high voltage circuit breaker were used for online monitoring and diagnosis.

\subsection{Feature extraction based on fast Fourier transform (FFT)}

As a signal analysis tool, Fourier transform can decompose and synthesize signals, and its formula is:

$$
F(\omega)=\int_{0}^{t_{1}} f(t) e^{-i \omega t} d t
$$

where $f(t)$ is the original signal before transformation, $F(\omega)$ is a transformed signal, $e^{i \omega t}$ is a complex variable function, $t$ is time, and $t_{1}$ is the signal duration. In essence, Fourier transform transforms the change rule of signal distributed in time domain into that of signal distributed in frequency domain, which is equivalent to making statistics on the frequency distribution of the whole analysis signal and cannot reflect the local time-frequency characteristics of the signal. But for the non-stationary vibration in this paper, the local time-frequency characteristics of the signal are the key classification basis. Short-time Fourier transform is also called windowed Fourier transform, and its formula $[8]$ is:

$$
W f_{g}(b, \omega)=\int_{0}^{t_{1}} f(t) g(t-b) e^{-i \omega t} d t
$$

where $W f_{g}(b, \omega)$ is the information transformed in the time window, $g(t-b)$ is a window function, and $b$ is a time translation factor. After adding the window function, compared with the previous Fourier transform, it has been partitioned on the time axis, which achieves local analysis.

\subsection{Feature extraction based on energy entropy of wavelet packet}

As mentioned above, the developed short-time Fourier transform can achieve local analysis of non-stationary signals due to the partitioning of time axis by window function. However, due to the fixed size and shape of time window, it is still full-frequency domain analysis in frequency domain and cannot fully display the fine signals in a certain frequency band at a single time point.

Wavelet transform represents or approximates the analytic signal using uses the system of wavelet functions, and its transformation formula [9] is:

$$
W_{f}(a, b)=\int_{0}^{t_{1}} f(t) \psi_{a, b}(t) d t
$$

where $W_{f}(a, b)$ is a transformed signal, $\psi_{a, b}(t)$ is a wavelet basis function, and $a$ and $b$ are the scale factor and translation factor of wavelet transform respectively. After transforming the detected signal with the wavelet transform formula mentioned above, the multi-resolution analysis of the signal can be carried out, and more accurate features can be obtained. However, the wavelet transform follows the binary variation. When the signal is processed, it only decomposes the low-frequency part of the signal, but does not process the high-frequency part. If the effective features of the signal are in the highfrequency part, it is difficult to obtain the effective features after the wavelet transform. In order to solve the above problems, the wavelet packet method is used to decompose the signal.

As shown in Figure 1, the wavelet packet after decomposition is:

$$
\begin{aligned}
S= & A A A 3+D A A 3+A D A 3+D D A 3+A A D 3 \\
& +D A D 3+A D D 3+D D D 3,
\end{aligned}
$$

where $A$ stands for low frequency and $D$ stands for high frequency. Hilbert method is used to extract the envelope signal from the three-level decomposition components. Then the envelope signal is divided into $\mathrm{n}$ parts according to the time. The characteristic entropy is calculated and used as the eigenvalue for recognition. 


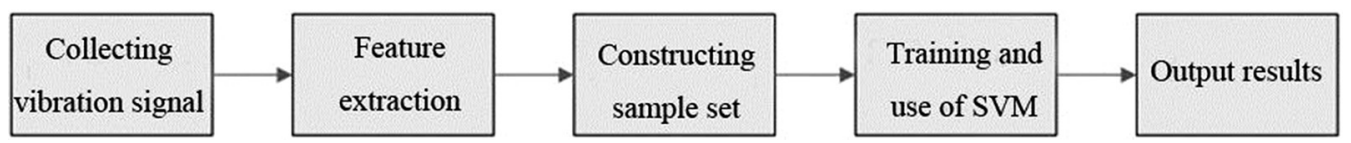

Fig. 2. The flow of circuit breaker fault detection based on SVM.

The formula is [10]:

$$
\left\{\begin{array}{l}
Q_{i}=\int_{t_{0}}^{t_{i}}|A(t)|^{2} d t \\
\varepsilon(i)=Q_{i} / \sum_{i=1}^{n} Q_{i} \\
H=-\sum_{i=1}^{n} \varepsilon(i) \log \varepsilon(i)
\end{array}\right.
$$

where $Q_{i}$ is the energy of the $i$-segment envelope signal, $A(t)$ stands for envelope signal, $t_{0}$ and $t_{i}$ are the starting and stopping time of the ith segment envelope signal respectively, $\varepsilon(i)$ is the result of the $i$ th envelope signal energy after normalization processing, and $H$ is the characteristic entropy of envelope signal.

\section{SVM algorithm}

SVM algorithm [11] is an intelligent algorithm which can learn by itself. Its basic principle is to project the data into the high-dimensional space using kernel function, then find the optimal classification boundary according to the principle of linear partition, and classify the detection data according to the classification boundary. Ideally, the sample used for training or testing has the property of linear separability and can find the optimal boundary which divides the data into two categories. But in practice, the sample data often does not have the property of linear separability. Therefore, the slack variable [12] and penalty parameter are introduced. Then the objective function for solving the problem is:

$$
\min _{w, b} \frac{\|w\|^{2}}{2}+C \sum_{i=1}^{l} \xi_{i}
$$

the condition is:

$$
y_{i}\left(w \cdot x_{i}+b\right) \geq 1-\xi_{i}
$$

where $w$ stands for weight, $C$ is penalty parameter, $\xi_{i}$ is a slack variable, $y_{i}$ is the result of classification, $x_{i}$ is the sample data, and $b$ is the residual term. Then the Lagrange function [13] and dual theory are used to transform the solution function into:

objective function:

$$
\max Q(a)=\sum_{i=1}^{l} a_{i}-\frac{1}{2} \sum_{i=1}^{l} \sum_{j=1}^{l} a_{i} a_{j} y_{i} y_{j} K\left(x_{i}, x_{j}\right),
$$

condition:

$$
\sum_{i=1}^{l} a_{i} y_{i}=0 \quad 0 \leq a_{i} \leq C,
$$

where $a$ is the set of $a_{i}, a_{i}$ is the Lagrange coefficient, $l$ is the sample size, and $K\left(x_{i}, x_{j}\right)$ is the kernel function [14]. Finally, the decision equation is obtained:

$$
f(x)=\operatorname{sgn}\left(\sum_{i=1}^{l} a_{i} y_{i} K\left(x_{i}, x_{j}\right)+b\right) .
$$

As shown in Figure 2, the detection process for high voltage circuit breaker faults is as follows:

- Vibration signal of circuit breaker is collected by vibration sensor during operation, including in normal working state, loosening state of fixed screw and state of falling of opening spring.

- The methods proposed above are used to extract the features of vibration signals.

- After signal feature extraction, sample set is constructed by using the extracted feature set, and one part of the sample set is used as training sets and the other part is used as testing sets.

- Training sets are into SVM, and appropriate $K\left(x_{i}, x_{j}\right)$ and $C$ are set. The objective function is solved to obtain the weight of optimal boundary, $w$, and the residual term, $b$. Finally the decision function is obtained. At this moment, the SVM training finishes.

- The testing sets are input into the SVM for fault type classification.

\section{Experimental analysis}

\subsection{Experimental platform}

As shown in Figure 3, the experimental platform for detecting high-voltage circuit breakers consists of highvoltage circuit breakers, vibration sensors, level conversion circuits, AD conversion modules, signal processing modules and upper computer system. The feature extraction algorithm and SVM algorithm are compiled by MATLAB software [15]. In the SVM algorithm, the radial basis function is chosen as the kernel function, and the penalty parameter is 0.01 .

\subsection{Experimental steps}

- The vibration sensor is adsorbed on the surface of the circuit breaker mechanism box by magnet. 


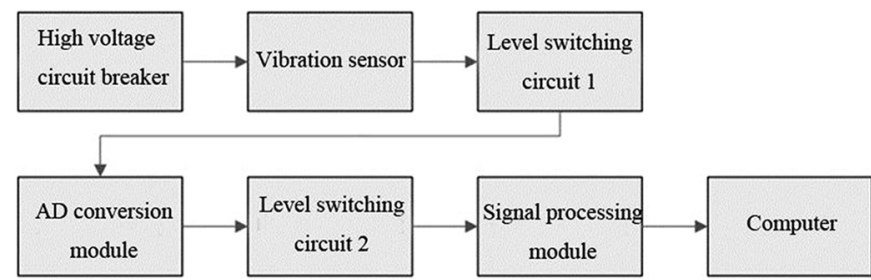

Fig. 3. Structure diagram of fault circuit breaker detection.

- High voltage circuit breaker is used to simulate three working states: normal operation, loosening of fixed screw and falling of opening spring. Vibration information of circuit breaker is collected by vibration sensor.

- Vibration signals of circuit breakers under three working conditions were collected for $12 \mathrm{~s}$, and each $0.06 \mathrm{~s}$ was taken as a sample. There were 200 samples in each working condition, among which $60 \%$ of the samples were taken as training set and $40 \%$ as testing set.

- Four methods, including Fourier transform, short-time Fourier transform, wavelet transform and energy entropy of wavelet packet, are used to extract features from training sets, and SVM is input into the fault detection model for training.

- After the training of the fault detection model, the performance of the model is tested by using the testing set.

\subsection{Performance index}

In this study, classification result is represented by the confusion matrix, and its expression is:

$$
N=\left[\begin{array}{ccc} 
& A & B \\
A & T P & F N \\
B & F P & T N
\end{array}\right] .
$$

Based on matrix (10), the performance of the algorithm is measured by accuracy, false alarm rate and precision rate. The calculation formula is:

$$
\left\{\begin{array}{l}
A C C=\frac{T P+T N}{T P+T N+F P+F N} \\
F A R=\frac{F N}{T P+F N} \\
D R=\frac{T P}{T P+F P}
\end{array},\right.
$$

where $A C C, F A R, D R$ stand for the accuracy rate, false alarm rate and precision rate, $T P$ stands for the number of faults which are classified as $A$ and actually belong to $A$, $T N$ stands for the number of faults which are classified as $B$ and actually belong to $B, F P$ stands for the number of faults which are classified as $B$ and actually belong to $A$, and $F N$ stands for the number of faults which are classified as $A$ and actually belong to $B$.

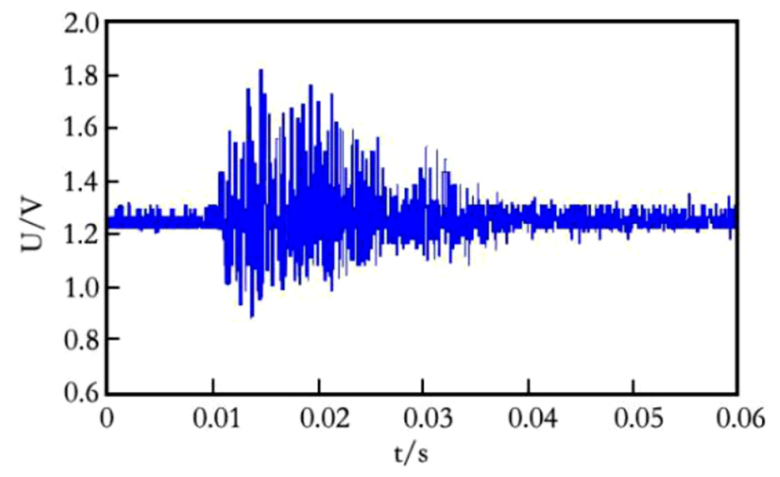

Fig. 4. The vibration waveform when the circuit breaker operates normally.

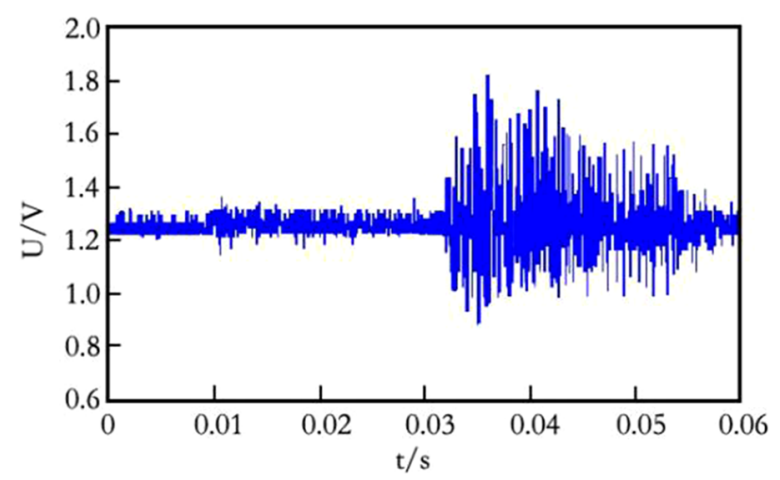

Fig. 5. The vibration waveform when the fixed screw loosens.

\subsection{Experimental results}

There are totally 600 vibration information of circuit breakers in different working states collected by vibration sensors. Through naked eye observation, it is found that the vibration waveforms of circuit breakers in the same working state are similar in general. Limited by the length of paper, only one vibration waveform in each working state is displayed. The vibration waveforms of high voltage circuit breakers in the normal working state are shown in Figure 4. It can be found from Figure 4 that there is a vibration with a large amplitude in a short time when the circuit breaker operates normally and then the amplitude gradually decreases. The sudden increase of the amplitude in a short time is because that the circuit breaker needs to react quickly when it perceives the fault of the power grid and the force and speed during closing are large. The decrease of the amplitude is because that there are no other actions after closing and the vibration decreases under the action of resistance. Figure 5 shows the working vibration waveform when the fixed screw of the circuit breaker is loosened. Compared with the vibration waveform under the state of normal working, it is found that the waveform is close, but the occurrence is delayed. It shows that the fixed screw can not react quickly although it can still be switched on after loosening. Once the power grid fails, the circuit breaker in this state cannot be switched on quickly because of loosing, and the loss may be enlarged in that 


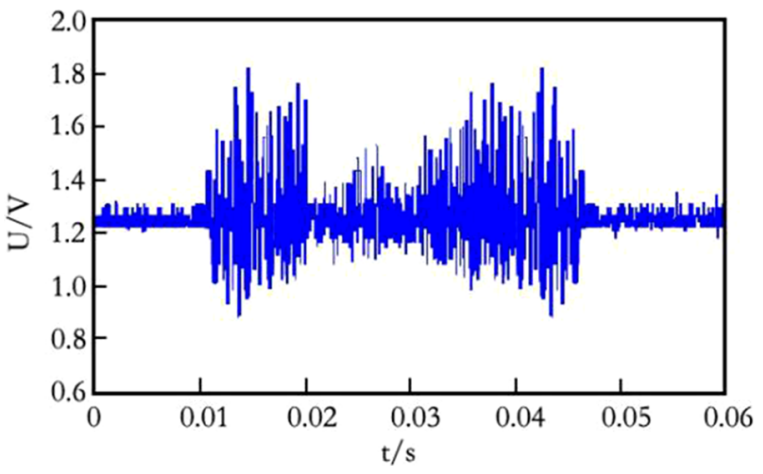

Fig. 6. The vibration waveform when the opening springs falls off.

period. Figure 6 shows the vibration waveform when the opening spring of the circuit breaker falls off. The time when the vibration appears is close to the vibration in the normal state, but the waveform is obviously different. In such a fault state, the circuit breaker will make switching operation quickly when it detects the fault of the power grid, but the circuit break fails to completely close because of the falling of the opening spring, leading to the decrease of the vibration amplitude. After that, the closing is attempted constantly, and the vibration amplitude increases.

It can be found from Figures 4-6 that the vibration waveforms of high voltage circuit breaker after the appearance of fault are quite different from those without the appearance of fault, and the difference was significant. The naked eye can indeed distinguish different faults, but in practical application, the coverage of power grid system is large and the number of high voltage circuit breakers is large. Though some detection time can be saved through vibration signals can be summarized to the head server by wireless sensor, the enormous signal data cannot be processed manually. In addition, once the breaker fault is detected, it is necessary to make a judgment as soon as possible in order to prevent the harm from expanding. Manpower observation can not make a quick judgment in large data volume, so it is necessary to use SVM algorithm and the fast processing ability of computer to assist fault monitoring.

The recognition accuracy rates of four feature extraction methods are shown in Figure 7 . It can be seen intuitively that no matter in what kind of working state, the fault detection model which extracts features by wavelet packet energy entropy has the highest recognition accuracy rate, followed by wavelet transform feature extraction, short-time Fourier transform feature extraction and Fourier transform feature extraction, indicating that the feature extracted by wavelet packet energy entropy method is more accurate. By comparing the accuracy rate of the model based on the same feature extraction method in recognizing faults under different working states, it can be found that the recognition effect of the recognition model is similar, but the recognition for the normal working state is slightly better.

The false alarm rates of the four feature extraction methods are shown in Figure 8. It can be seen that the false

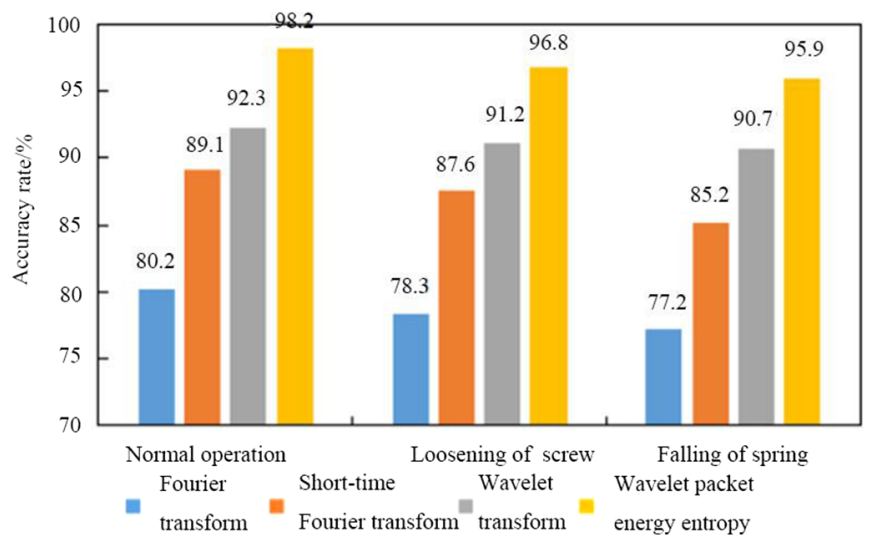

Fig. 7. Recognition accuracy rate of the fault detection model based on different feature extraction methods.

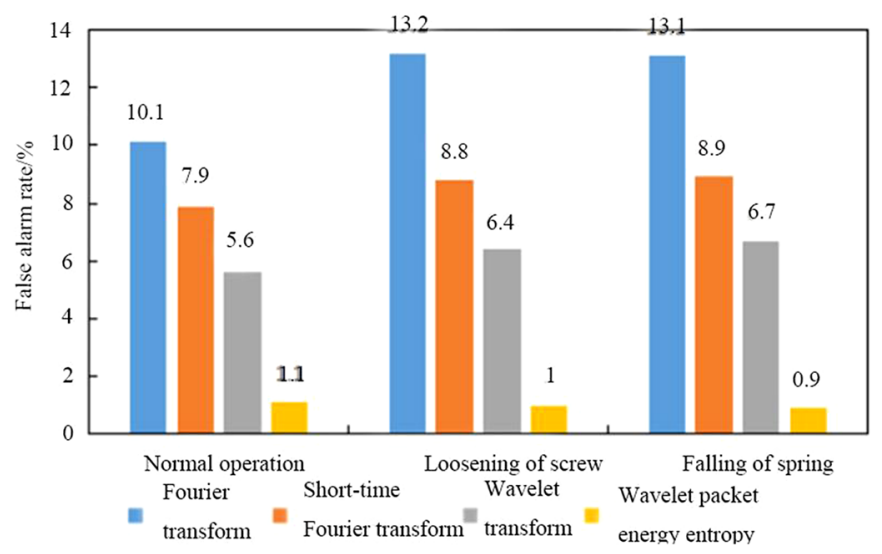

Fig. 8. Recognition false alarm rate of the fault detection model based on different feature extraction methods.

alarm rates of the four feature extraction methods are low when detecting faults under the normal working state and high when detecting faults under the other two states, and moreover the false alarm rate of the feature extracted by the energy entropy of wavelet packet is the lowest in any working state.

As shown in Figure 9, the precision rate is $70.2 \%, 72.3 \%$ and $78.2 \%$ in the normal state, state of loosen screw and state of falling of spring when of Fourier transform is used, $85.15 \%, 86.4 \%$ and $86.3 \%$ when short-time Fourier transform is used, $88.32 \%, 92.5 \%$ and $89.4 \%$ when wavelet transform is used, and $99.1 \%, 97.3 \%$ and $96.7 \%$ when the energy entropy of wavelet packet is used. It can be seen that the precision rate is the highest no matter under what kind of state when the features extracted by the energy entropy of wavelet packet are used, and moreover the difference is not significant. The precision rate of the model based on the other three methods has fluctuations under different operation states.

It can be seen from Figures 7-9 that the feature detection based on wavelet packet energy entropy had higher accuracy and precision and lower false alarm rate in detecting the normal circuit breaker and circuit breaker with two kinds of faults. The reason is that Fourier 


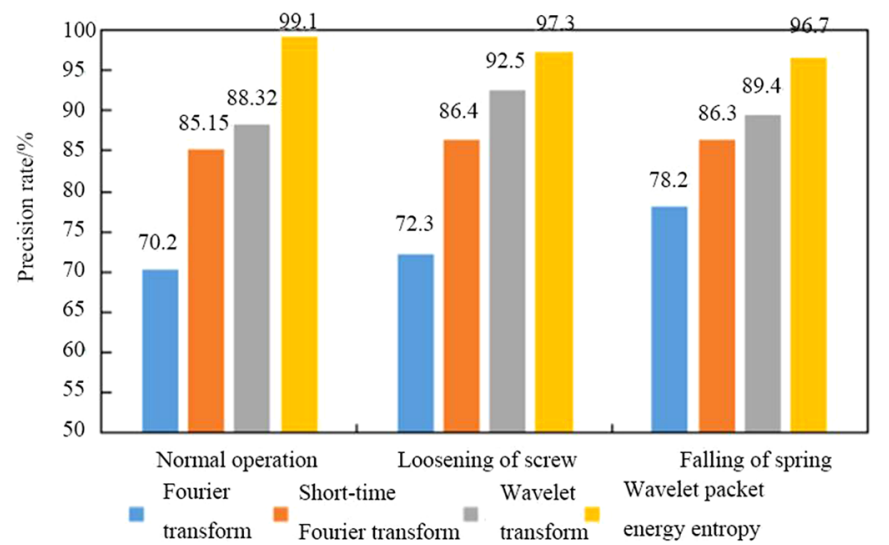

Fig. 9. Recognition precision rate of the fault detection model based on different feature extraction methods.

transform transforms the variation rule of signal distributed in time domain into the variation rule of signal distributed in frequency domain, which is equivalent to making statistics on the frequency distribution of the whole analysis signal and cannot reflect the local time-frequency characteristics of the signal. However, the vibration signal detected in this study is non-stationary, and its main features are concentrated in local position. Short-time Fourier transform (STFT) alleviates the original problem to some extent. The added time window function partitions the time axis of the signal and reflects the local timefrequency characteristics to some extent. However, due to the fixed size and shape of the time window, it is still fullfrequency domain analysis in the frequency domain, which cannot fully display the fine signal in a certain frequency band in a single time point. After the wavelet transform formula is applied to the detection signal, the multiresolution analysis of the signal can be carried out, and more accurate features can be obtained. However, the wavelet transform follows the binary variation. When the signal is processed, it only decomposes the low-frequency part of the signal, but does not process the high-frequency part. The effective features of the vibration signal are in the high-frequency part, and it is difficult to obtain the effective features after the wavelet transform. Wavelet Packet Transform divides the signal into low frequency and high frequency and made multi-scale decomposition of wavelet transform, so as to obtain more complete signal features. The more complete and accurate the extracted features are, the higher the accuracy and accuracy of SVM training and detection and the lower the false alarm rate.

The recognition time of the four feature extraction methods is shown in Figure 10. It can be seen that the application of different feature extraction methods will affect the recognition time of the fault recognition model. The recognition time of the detection model based on wavelet packet energy entropy is the shortest, and the recognition time of the model based on Fourier transform is the longest. The results show that feature extraction based on wavelet packet energy entropy can significantly improve the recognition efficiency of the recognition model.

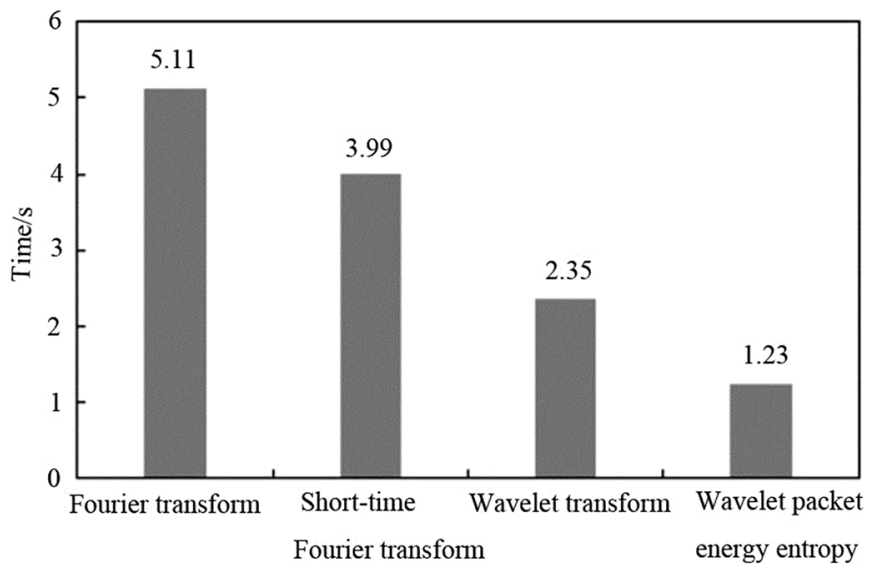

Fig. 10. Recognition time of different feature extraction normal fault detection model.

\section{Conclusion}

This paper briefly introduces the feature extraction method of the vibration signal of high voltage circuit breaker and SVM algorithm and analyzed the high voltage circuit breaker in three states: normal operation, fixed screw loosening and falling of opening spring, using the SVM algorithm based on the above feature extraction method. The results are as follows. Vibration signals under three states are compared and analyzed, and the difference between them is obtained. Under three working states, the recognition rate of the model based on wavelet packet energy entropy feature extraction is the highest, followed by wavelet transform feature extraction, shorttime Fourier feature extraction and Fourier transform feature extraction; the recognition precision rate is the same, while the false alarm rate is the opposite. In the aspect of recognition time, the recognition time of the model based on Fourier transform feature extraction is the longest, followed by short-time Fourier transform, wavelet transform and wavelet packet energy entropy. The novelty of this study is that it realized the rapid and accurate determination on whether high voltage circuit breaker has faults and the types of faults through combining vibration feature extraction method with SVM algorithm and moreover compared different feature extraction methods.

\section{References}

1. K. Niayesh, Int. Tran. Electr. Energy Syst. 25, 1100-1112 (2015)

2. S. Zubić, M.B. Djurić, C. Zeljković, Electr. Power Syst. Res. 119, 218-227 (2015)

3. N.T. Huang, H.J. Chen, S.X. Zhang, G.W. Cai, W.G. Li, D.G. Xu, Entropy 18, 7 (2015)

4. L. Yang, Y. Zhu, Power Syst. Protect. Control 43, 62-67 (2015)

5. B. Li, M. Liu, Z. Guo, Y. Ji, Entropy 20, 448 (2018) 
6. N. Huang, L. Fang, G. Cai, D. Xu, H. Chen, Y. Nie, Entropy 18, $322(2016)$

7. X. Kong, H.J. Liu, Y.Z. Xie, J. Guo, Q. Liu, Y. Chen, S. Wang, X. Sun, IEEE Tran. Power Del. 31, 1168-1175 (2016)

8. Y. Zhao, P. Geng, C. Xu, J. Song, High Voltage Apparat. 53, 39-46 (2017)

9. J. Liu, N. Tai, C. Fan, S. Chen, IEEE Trans. Ind. Electr. 64, $1-1(2017)$

10. B. Chang, O. Cwikowski, M. Barnes, R. Shuttleworth, A. Beddard, P. Coventry, High Voltage 2, 211-219 (2018)
11. T. Ohtaka, V. Kertész, R.P.P. Smeets, IEEE Trans. Power Del. 33, 1-1 (2017)

12. X.G. Wu, X. Wang, Y. Zhu, C. Lin, D. Liu, W. Wang, High Voltage Eng. 43, 1079-1085 (2017)

13. S. Li, L. Geng, Y. Zhong, H. Han, High Voltage Apparat. 51, 84-87 (2015)

14. Y. Feng, J. Wu, C. Wang, D. Li, J. Yang, Y. Sun, High Voltage Apparat. 53, 1-7 (2017)

15. A. Rostami, A. Jalilian, M.T. Hagh, K.M. Muttaqi, J. Olamaei, IEEE Trans. Ind. Appl. 55, 954-963 (2018)

Cite this article as: Long Li, Jianfeng Xiao, Bin Wu, Mengge Zhou, Qian Wang, Online monitoring and diagnosis of high voltage circuit breaker faults: feature extraction analysis of vibration signals, Int. J. Metrol. Qual. Eng. 10, 13 (2019) 